\title{
Experimental study of a hybrid solar photovoltaic, thermoelectric and thermal module
}

\author{
Flávio Matias ${ }^{1}$, Luís C. Pires ${ }^{1,2,}$, Pedro D. Silva ${ }^{1,2}$, and Pedro D. Gaspar ${ }^{1,2}$ \\ ${ }^{1}$ University of Beira Interior, Rua Marquês d'Ávila e Bolama, 6201-001, Covilhã, Portugal \\ ${ }^{2}$ C-MAST - Centre for Aerospace Science and Technologies, 6201-001, Covilhã, Portugal
}

\begin{abstract}
Nowadays, solar energy, which can be photovoltaic and thermal, is a clean and reliable source of energy for the production of electric and thermal power. However, new ways for improving photovoltaic efficiency are fundamental for an extensive application of this technology. Most of the energy absorbed by the PV panel converts itself into heat, which usually is lost and does not have any energetic value. The performance of a combined photovoltaic (PV), thermoelectric generator (TEG) and water heating panel is tested in practice. The thermoelectric set is applied on the back of the PV panel so that the two devices have approximately the same temperature. On the other face of the thermoelectric set, there is the water heating panel, which consists of an aluminium heat exchanger specially designed for this hybrid module. The exposed surface of the hybrid panel has an area of about $2.72 \mathrm{dm}^{2}$. Experimental tests were conducted in direct solar exposure during July. The experimental results indicate that the maximum global module efficiency was $91.3 \%$ for an irradiance of $1089 \mathrm{~W} / \mathrm{m}^{2}$. The power peak production was $29.7 \mathrm{~W}$, at 2 p.m., with an irradiance value of $1230 \mathrm{~W} / \mathrm{m}^{2}$.
\end{abstract}

\section{Introduction}

Traditional photovoltaic panels convert only 6 to $15 \%$ of all incident energy. Most of the radiation converts into heat, which results in a higher operation temperature, leading to lower photovoltaic efficiency. In this case, all the thermal energy is wasted to the environment without any energy benefit. There are many solutions to minimise the temperature increase that reduces the photovoltaic panel performance, as shown by Mays et al. [1]. These researchers have studied the implementation of an aluminium plate with fins on the back of the PV panel, to dissipate a large amount of heat. In the end, they could achieve a performance increase of $1.77 \%$ on electrical energy and a reduction of $6.1{ }^{\circ} \mathrm{C}$ on the PV temperature. More works were done on this area involving PV effect and thermal energy, as the study of the Noro et al. [2] where they have studied the integration of photovoltaic-thermal systems of buildings. On that article, the work developed focused not only on $\mathrm{PV} /$ thermal systems but also on concentrating $\mathrm{PV} / \mathrm{T}$ systems and photovoltaic-thermal heat pumps systems. Various designs were applied, being the liquid the most effective cooling system, which provides a more homogenous temperature distribution on the PV surface. A similar work developed by Jie Ji et al. [3] focused on the material of the hybrid modules to achieve higher efficiency. Ultimately, they have indicated that the higher the PV cell covering factor and the glazing transmissivity, the better the overall performance. On the experimental setup, they could achieve an electrical efficiency of $10.15 \%$ and a thermal efficiency that exceeds $45 \%$, with a PV cell covering factor equal to 0.63 and a front-glazing transmissivity of 0.83 . A most recent work, conducted by Yang and Yin [4], provides not only electrical power but also hot water for heating purposes. These two investigators concluded that the hybrid module with PV panel and hot water supply provides more 30 to $50 \%$ on photovoltaic cells efficiency. The second model, which incorporates the thermoelectric elements, can recover 25 to $40 \%$ on PV panel efficiency. The main conclusion is that the system efficiency will increase when the TE technology reaches higher values of merit figure, leading to better performance on the thermoelectric modules. Kalogirou and Tripanagnostopoulos [5] proposed a more simply hybrid module, including only the photovoltaic effect and thermal energy. These two researchers demonstrated that the hybrid system with polycrystalline solar cells has a better performance when compared with the amorphous ones. A non-hybrid PV system produces about $38 \%$ more electrical energy but zero thermal energy. As a general conclusion, the overall energy production of the hybrid unit is increased, having a better chance of success, covering the hot water demands of a house, with a water storage tank of 160 litres. Chen et al. [6] provided a numerical study on the electrical performance of a PV panel cooled by natural ventilation for different values of solar radiation, air temperature and wind velocity. To improve heat dissipation from the PV panel an extended surface with metal fins on the back face of the panel was considered. Therefore fin size

Corresponding author: pires@ubi.pt 
effect on PV energy performance was also evaluated. The main conclusions were that higher solar radiation intensities and higher wind velocities led to better electrical performance of the PV panel. On the other hand, the electrical efficiency linearly decreased with the increase on the air temperature. In all evaluated conditions the electrical efficiency of PV panel with fins was higher than that of PV panel without fins. In a complementary study to this one Chen et al. [7] conducted an experimental study with the same major goals of the previous one. The conclusions are in line with those of the previous study, either for temperature variation, wind velocity variation or solar radiation intensity variation. For the various imposed conditions of the study the average electrical efficiency of PV panel with fins was 0.3 to $1.8 \%$ higher than that of PV panel without fins and the average power output of PV panel with fins was 1.8 to $11.8 \%$ higher than that of PV panel without fins. A photovoltaic thermal hybrid system was also studied by Chao-Yang and Chiou-Jye [8] who created a model using TRNSYS to simulate transient and long term evaluation of the hybrid system to predict its performance in different weather conditions. The study was based on a real system consisting of six PVT collectors with total exposed area of $9.78 \mathrm{~m}^{2}$ and a 5001 water storage tank. The simulation results have shown electrical efficiencies from 11.7 to $12.4 \%$ and a thermal efficiency from 26.78 to $28.41 \%$. Another system designed for extracting more power of the PV panels consists of the inclusion of thermoelectric elements, as done by Zhu et al. [9] on a numerical investigation. This team of investigators have studied the overall efficiency on a PV module with thermoelectric technology placed on the back of the PV cell. The results prove that the new hybrid module has a $25 \%$ better efficiency than just the PV cell. One theoretical work, conducted by Bjørk and Nielsen [10], proposed a combined system where the thermoelectric elements are mounted directly on the back of the PV panel, and a tandem system, where the incoming radiation is split, with the short wavelength radiation sent to the PV and the long-wavelength delivered to the thermoelectric set. The maximum increase in efficiency was $4.5 \%$ for the combined case and only $1.8 \%$ for the tandem design. An experimental investigation was done by Soltani et al. [11], about the cooling methods for a hybrid photovoltaic/thermoelectric system. The highest power and efficiency reached on the $\mathrm{SiO}_{2} /$ water nanofluid was respectively $54.29 \%$ and $3.35 \%$. Water cooling method produced $47.7 \%$ more power compared with the natural cooling method, which was the natural air circulation.

A hybrid solar photovoltaic, thermoelectric and thermal module shall be presented in this paper. The purpose of combining those three technologies was to harness the usual lost thermal energy, to provide hot water supply, and adding some electric production by placing a thermoelectric generator between PV panel and water heater, taking advantage of the correspondent temperature differences. Hence, the thermoelectric set and the PV panel will produce electrical energy, and simultaneous the water heater will produce thermal energy.

\section{Material and methods}

A schematic of the testing unit is shown in Figure 1, with all the equipment needed to conduct this practical experience. Figure 2 shows the assembly process inside the module, indicating each layer that compounds the hybrid module. Around all the constituent elements, shown in Figure 2, it was attached a box made of extruded polystyrene taking advantage of its excellent insulating propriety. The top of the PV panel is coincident with the top surface of the thermal insulating box, allowing to receive direct radiation from the sun, without any shadows.

\subsection{Materials}

The experimental hybrid module was fully built and assembly for this experiment. The hybrid module is compound by a PV panel, a thermoelectric generator set with 16 TEC cells and a customised aluminium water heater panel that aims to the hot water production. First, the most crucial phase was the choice and purchase of all the items to building the hybrid module because all the dimensions must be coincident, or at least as closely as possible. At the bottom of the hybrid module, there is the water heater panel, made of aluminium alloy 6082 T451, with high thermal conductivity (145 to $165 \mathrm{~W} / \mathrm{mK}$ ). The water heater panel has a square shape, with dimension sides of $0.165 \mathrm{~m}$, and it was specially designed for this module. Two plates make this thermal element with a thickness of $0.005 \mathrm{~m}$ each, then when close totalize a thickness of $0.01 \mathrm{~m}$. Each plate has a circuit with square channels that will allow the water flow, and these channels have $0.01 \mathrm{~m}$ of width. This internal fluid circuit has 1.285 meters inside the aluminium

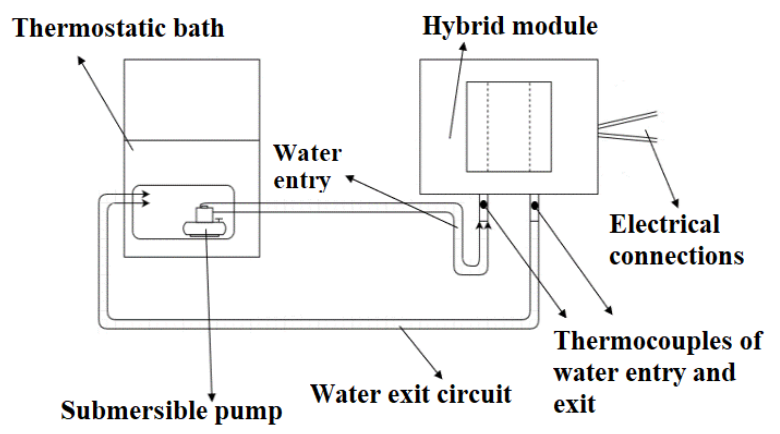

Fig. 1. Testing an installation scheme.

The next and upside compound is the thermoelectric set. This part contains 16 thermoelectric coolers (TEC) used in this work as thermoelectric generators (TEG). These elements have been divided into four sectors, electrical connections in series each sector have four cells, also connected in series. The choice of the TEC model was taken by the previous work of Maneewan and Chindaruksa [12]. On this work, the generator performance of the TEC1-12708 was superior on lower temperatures, and this kind of thermoelectric modules are cheaper. These thermoelectric modules have 127 pairs of thermoelectric elements each, which are 
responsible for the electrical production. This kind of thermoelectric cell has two plates of ceramic, and one layer also has the serial number indicating the hot face of the module. In the building process of this thermoelectric set, we have included thin pieces of cork to reduce the heat conduction between the hot and cold faces of each TEC. Table 1 presents the main features of the thermoelectric cells used in this work.

Table 1. Technical data of the TEC cells.

\begin{tabular}{ccc}
\hline Parameter & Symbol & Value \\
\hline Manufacture & - & TMOEC \\
Model & - & TEC1-12708 \\
Dimensions & $\mathrm{mm}$ & $40 \times 40 \times 3.5$ \\
Thermoelectric elements & - & 127 \\
Working voltage & $\mathrm{V}$ & 15.4 \\
Working current & $\mathrm{A}$ & 8.5 \\
Max. working temperature & ${ }^{\circ} \mathrm{C}$ & 138 \\
Length of the elect. & $\mathrm{mm}$ & 250 \\
conductors & & \\
Internal resistance & $\Omega$ & 1.3 to 1.7 \\
Max. heat dissipation & $\mathrm{W}$ & 74 \\
$\Delta$ T maximum & ${ }^{\circ} \mathrm{C}$ & 138 \\
\hline
\end{tabular}

Between the water heater module and the thermoelectric set, two layers of one thermal conductor were applied. This particular layer has the purpose of improving the heat transmission between the water heater panel and the thermoelectric modules. The material used behaves like a sponge that is compressed between the two surfaces.

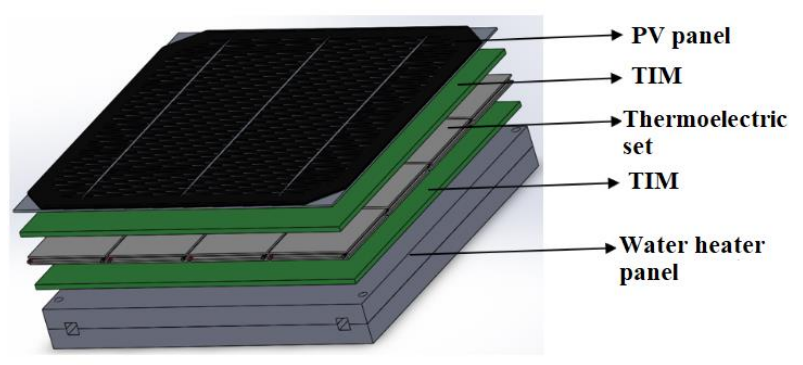

Fig. 2. Assembly experimental setup.

At the top of the hybrid module there is the photovoltaic panel with a dimension of 0.165 by 0.165 meters, which makes an area of about $2.72 \mathrm{dm}^{2}$. This monocrystalline panel has $7 \mathrm{~V}$ open-circuit voltage and $720 \mathrm{~mA}$ short circuit current, and it has been selected because of its optimal dimensions that perfectly match with the thermoelectric set and the water heater panel. Underneath the PV module, we have applied a new layer of a thermal interface material (TIM) to reduce the thermal contact resistance between the PV module and the set of thermoelectric. Table 2 presents the major technical features of the PV panel used.
Table 2. Technical data of the PV panel.

\begin{tabular}{ccc}
\hline Description & Symbol & Specification \\
\hline Manufacture & - & STAR SOLAR \\
Model & - & CNC $165 \times 165-6$ \\
Dimensions & $\mathrm{mm}$ & $165 \times 165 \times 2$ \\
Type of cells & - & Monocrystalline \\
Open voltage & $\mathrm{V}$ & 7.2 \\
Short circuit current & $\mathrm{A}$ & 0.850 \\
Work voltage & $\mathrm{V}$ & 6.0 \\
Work current & $\mathrm{A}$ & 0.750 \\
Nominal power & $\mathrm{W}$ & 4.5 \\
\hline
\end{tabular}

The support material was used around the complete set, also ensuring good thermal insulation of the assembly. The material used for that purpose was extruded polystyrene, with thermal conductivity of only $0.034 \mathrm{~W} / \mathrm{mK}$. In this way, we consider that there are neither heat gains nor losses from outside. The only thermal energy input considered was the one entering as solar radiation by the PV panel, through the thermoelectric system and finally reaches the water heater panel.

\subsection{Setup assembly}

At the same time, during this construction process, there was the placement of thermocouples, two for registering the PV panel temperatures, two for the measurement of the hot and cold temperatures of the thermoelectric set, and the last was placed underneath the water heater panel. All of the thermocouples used were of T-type. With the use of these small probes it is possible to know the instant temperature of each layer of the hybrid module.

The experimental setup was oriented to South, at $30^{\circ}$ with the horizontal, and the tests were conducted on a place without obstacles or shadows, which is a fundamental condition for correct photovoltaic installation. A thermostatic bath was used to determine the thermal energy extracted from the water heater. In this equipment, we have to define one target temperature and wait for that temperature to be reached on the amount of water contained inside the bath. After this process, the water may circulate to the water heater panel, on a well-defined temperature. The water flow is ensured by the employment of a small submersible pump of $5 \mathrm{~W}$. The volumetric flow rate was fixed and had a value of six litres per hour. In this way, and with the help of the two thermocouples placed on the entry and the exit of the water heater panel, we can calculate the thermal energy produced by this element.

\subsection{Data gathering process}

The intensity of solar irradiance, the interface temperatures, the electric currents and voltages values were measured in each experimental test every fifteen minutes. Also, the ambient temperature was measured at the same time break. The experiments were performed over four consecutive days, with climatic conditions 
partial unchanged. The maximum and minimum temperatures were obtained on the website of Portuguese Weather Agency. They also provide the wind speed for the city where the tests were performed, Covilhã (4016'57" North, $7^{\circ} 30^{\prime} 12^{\prime \prime}$ West). All the weather data are presented in Table 3 .

Table 3. Weather conditions.

\begin{tabular}{lccc}
\hline & $\mathbf{T}_{\text {MIN }}\left[{ }^{\mathbf{}} \mathbf{C}\right]$ & $\mathbf{T}_{\text {MAX }}\left[{ }^{\mathbf{0}} \mathbf{C}\right]$ & Wind speed $[\mathbf{m} / \mathbf{s}]$ \\
\hline Day 1 & 13 & 32 & 1.11 to 1.94 \\
Day 2 & 15 & 33 & 1.39 to 2.50 \\
Day 3 & 14 & 31 & 0.83 to 1.39 \\
Day 4 & 15 & 35 & 1.39 to 2.50 \\
\hline
\end{tabular}

As mentioned above, all variables were updated and duly noted every fifteen minutes. That includes seven temperatures (measured by the thermocouples of T-type with $0.05^{\circ} \mathrm{C}$ uncertainly), the irradiance value (measured by a digital solar meter) and the electrical currents and voltages from both electrical circuits (measured with digital multimeters with $0.005 \mathrm{~V}$ uncertainly).

\subsection{Power and efficiency of the hybrid module}

The principal parameters to be calculated are the power of each panel and, of course, the sum of the three power panels, photovoltaic, thermoelectric and thermal. Furthermore, the overall efficiency of the hybrid module was calculated. Equation (1) gives the electrical power calculation for the photovoltaic set, $P_{P V}$ [13]. The second equation, again for electrical power, $P_{T E C}$, is applied to the thermoelectric set [14].

$$
\begin{gathered}
P_{P V}=U_{P V} \times I_{P V} \\
P_{T E C}=U_{T E C} \times I_{T E C}
\end{gathered}
$$

Where $U_{P V}$ and $U_{T E C}$ are the voltages for the photovoltaic and thermoelectric panel, respectively, and $I_{P V}$ and $I_{T E C}$ are the electric currents for the photovoltaic and thermoelectric panel, respectively.

On the same way, the last power to be calculated is the thermal power delivered by the water heater panel, $\dot{Q}$. This calculation is presented on the equation number 3 [15].

$$
\dot{Q}=\dot{m} \times c_{p} \times \Delta T_{H_{2} O}
$$

Where $\dot{m}$ is the mass flow, $c_{p}$ is the specific heat of the water, and the last term is the difference of temperature between the escape and the entry of water on the aluminium heater panel.

The total power produced by the hybrid module is the sum of the previous equations

$$
P_{\text {hybrid module }}=P_{P V}+P_{T E C}+\dot{Q}
$$

The efficiency of the module is calculated by equation (5)

$$
\eta=\frac{P_{\text {OUT }}}{P_{I N}}=\frac{P_{\text {hybrid module }}}{E_{e} \times A}
$$

Where, $P_{\text {hybrid module }}$, represents the power of the module, $E_{e}$ is the irradiance value, and $A$ is the area of the module.

\section{Results and discussion}

The tests were conducted by successively incrementing the load value, beginning with no electrical loads on both electrical circuits. The following experiment, in the second day, had electrical loads on the PV circuit and TEC set, respectively of $21.9 \Omega$ and $3.4 \Omega$. The loads were increased to $56.6 \Omega$ and $10 \Omega$ on the third day, and on the fourth day the electrical charges were $101 \Omega$ and $21.9 \Omega$, for the PV panel and thermoelectric set, respectively. The power values produced by the hybrid module on these different electrical configurations are presented in Figure 3 and the solar irradiance values are presented in Figure 4.

\subsection{Power results}

In this section, the power is discussed for the four experiments conducted. The first one, without any electrical load, achieved $29.68 \mathrm{~W}$. This was the value for the power peak production by the hybrid module. The second test, now with $21.9 \Omega$ on the PV circuit and $3.4 \Omega$ on the thermoelectric set, came stable on the $22 \mathrm{~W}$. The third test, with middle loads (56.6 $\Omega$ for PV and $10 \Omega$ for TEC), was the worse test, with a power production lower than in the test with high loads (101 $\Omega$ for PV and 21.9 $\Omega$ for TEC), this significant difference is due to the irradiance values, that were also lower on the third day. These last two tests were accomplished with some power instability, with values between 10 and $17 \mathrm{~W}$.

\subsection{Irradiance results}

Figure 4 shows the amplitude on the irradiance of all four days; the values of the chart are very close on the first, second and fourth days. The third day, the one of middle loads (56.6 $\Omega$ for PV and $10 \Omega$ for TEC), was the day with minor sun irradiance. All the outside tests were likely influenced by clouds, which could be high clouds, with low influence on the PV panel, or lower and denser clouds, with more significant impact on the irradiance value availed on the PV surface. All measures of solar irradiance were made with a solar meter, with an uncertainty of $0.5 \mathrm{~W} / \mathrm{m}^{2}$. 


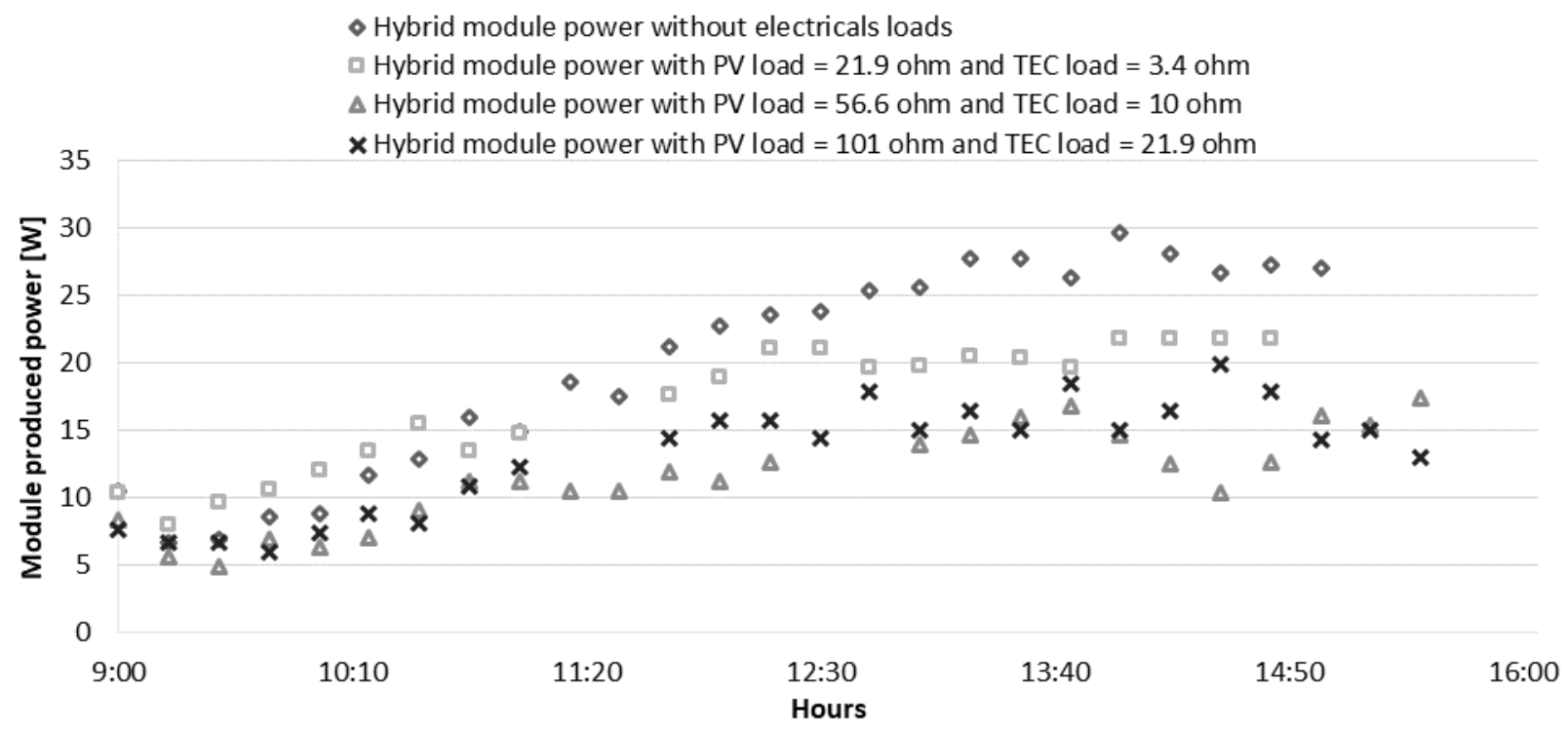

Fig. 3. Hybrid module produced power for different load values.

\subsection{Efficiency results}

The minimum hybrid module efficiency was $31.4 \%$, which is twice as high as the one of a typical monocrystalline. Efficiency results superior to one should be neglected, as they are caused by measurement errors probably associated with the thermal power determination.

As we can observe in Figure 5, the majority of the results are in the interval between 0.4 and 0.8 , being this last number a typical efficiency value for thermal solar panels. If the amount of incident power were $1000 \mathrm{~W} / \mathrm{m}^{2}$, which represents an amount of power entry on the PV surface equal to $27.2 \mathrm{~W} / \mathrm{m}^{2}$, this hybrid module would produce a total of $10.89 \mathrm{~W}$ to $21.78 \mathrm{~W}$ in electrical and thermal power. The thermal power represents 70 to $83 \%$ of the total power generated by the hybrid module.

\section{Conclusion}

In this experimental study, the production power and the efficiency of a hybrid module design consisting of a photovoltaic panel, a thermoelectric set and a water heater panel was investigated for four types of electrical loads (no load, low load, middle load and high load).The experiments were performed in four consecutive days, with quite identical atmospheric and climatic conditions, with negligible wind speed. The tests show that the efficiency of the hybrid module achieves, with some consistency, more than $80 \%$ of the overall efficiency. This value proves that hybrid modules can produce electrical and thermal energy with high efficiency. Unique PV panels can score only $13 \%$ of the performance, and the lowest practical value on this hybrid module was $33 \%$. The conclusion is that this hybrid module is $254 \%$ better, when compared to a typical monocrystalline PV panel, and provides both

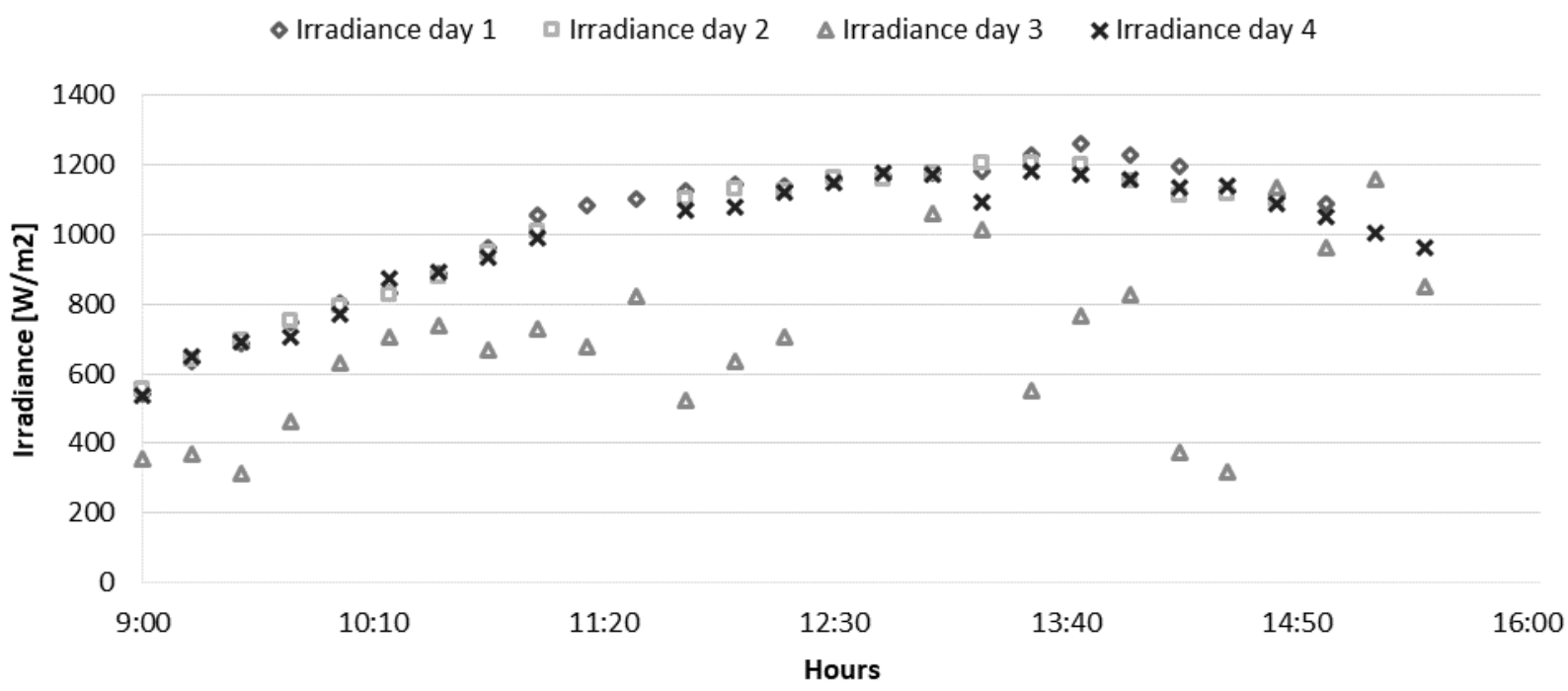

Fig. 4. Solar irradiance values of the four days. 


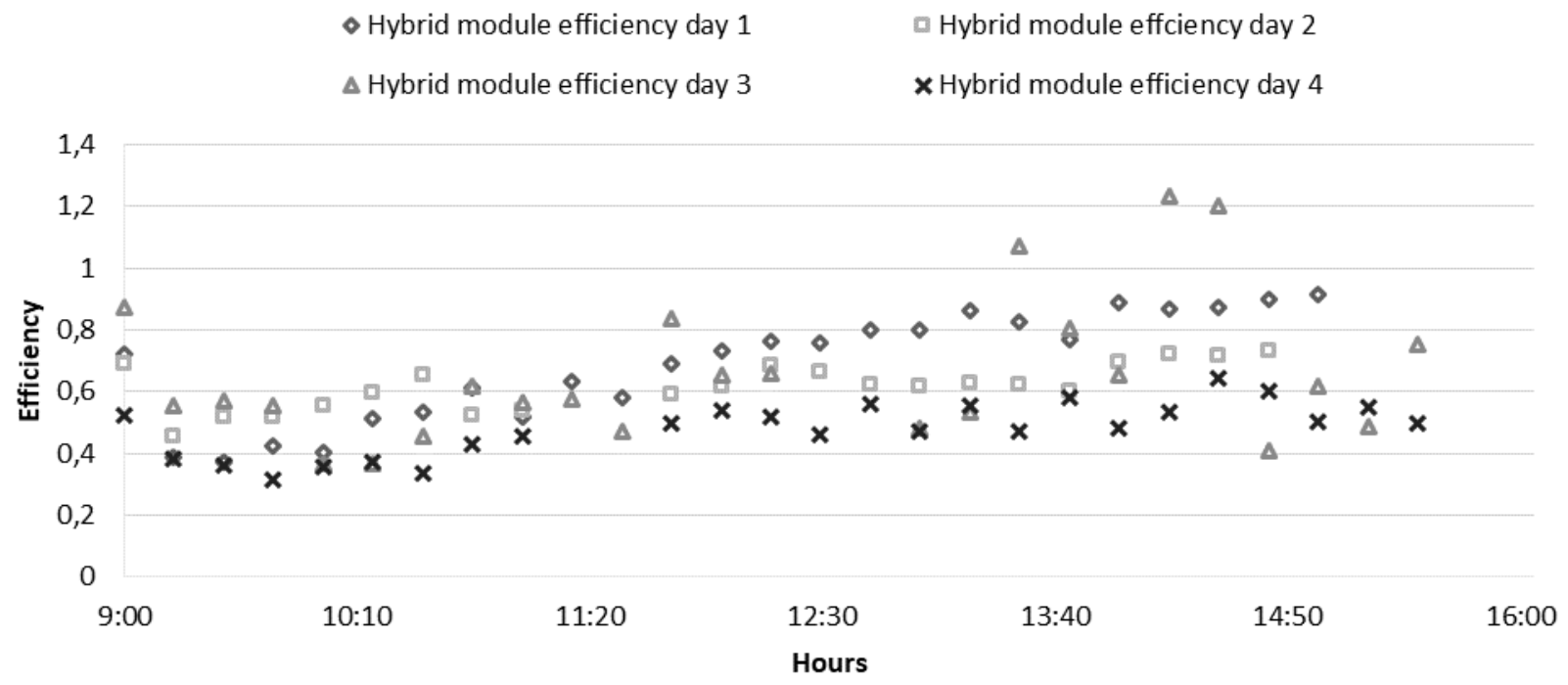

Fig. 5. Hybrid module efficiency for different load values.

electric and thermal energy. More experiments and search on this field are needed, to find a solution to the temperature rising on the photovoltaic panels. One solution could be, for example, the utilisation of latent heat technologies for hot water supply. The integration of two or more energetic technologies is the key to reduce the power losses and increment the global efficiency to values near the unit.

\section{References}

1. A.E. Mays, R. Ammar, M. Hawa, M.A. Akroush, F. Hachem, M. Khaled, M. Ramadan, Energy Procedia, Improving photovoltaic panel using a finned plate of aluminium, 119, 812-817 (2017)

2. M. Noro, R. Lazzarin, G. Bagarella, Energy Procedia, Advancements in hybrid photovoltaicthermal systems: performance evaluations and applications, 101, 496-503 (2016)

3. J. Ji, J. Lu, T. Chow, W. He, G. Pei, Applied Energy, A sensitivity study of a hybrid photovoltaic/thermal water-heating system with natural circulation, 84, 222-237 (2007)

4. D. Yang, H. Yin, IEEE Transactions of Energy Conversion, Energy conversion of a novel hybrid solar system for photovoltaic, thermoelectric, and heat utilization, 26, 662-670 (2011)

5. S.A. Kalogirou, Y. Tripanagnostopoulos, Energy conversion and management, Hybrid PV/T solar systems for domestic hot water and electricity production, 47, 3368-3382 (2006)

6. H. Chen, X. Chen, S. Li, H. Ding, Int. J. Smart Grid and Clean Energy, Numerical study on the electrical performance of photovoltaic panel with passive cooling of natural ventilation, 3, 395-400 (2014)
7. H. Chen, X. Chen, S. Li, H. Ding, Int. J. Smart Grid and Clean Energy, Comparative study on the performance improvement of photovoltaic with passive cooling under natural ventilation, 3, 374-379 (2014)

8. C.Y. Huang, C.J. Huang Int. J. Smart Grid and Clean Energy, A study of photovoltaic thermal (PV/T) hybrid system with computer modelling, 3, 75-79 (2014)

9. W. Zhu, Y. Deng, Y. Wang, S. Shen, R. Gulfam, Energy, High-performance photovoltaicthermoelectric hybrid power generation system with optimized thermal management, 100, 91-101 (2016)

10. R. Bjørk, K.K. Nielsen, Energy Conversion and Management, The maximum theoretical performance of unconcentrated solar photovoltaic and thermoelectric generator systems, 156, 264-268 (2018)

11. S. Soltani, A. Kasaeian, H. Sarrafha, D. Wen, Solar Energy, An experimental investigation of a hybrid photovoltaic/thermoelectric system with nanofluid application, 155, 1033-1043 (2017)

12. S. Maneewan, S. Chindaruksa, Journal of electronic materials, Thermoelectric power generation system using waste heat from biomass drying, 38, 974-980, (2009)

13. G.K. Singh, Energy, Solar power generation by PV (photovoltaic) technology: A review, 53, 1-13 (2013)

14. D. Enescu, F. Spertino, Applications of hybrid photovoltaic modules with thermoelectric cooling, 111, 904-913, (2017)

15. H.G. Teo, P.S. Lee, M.N.A.Hawlader, An active cooling system for photovoltaic modules, 90, 309315 (2012) 